\title{
High serum ferritin as a sensitive biomarker for diagnosis, follow up, prognosis and management of rheumatoid arthritis, brucellosis, malignancies, DM type 2 and vitamin b12 deficiency
}

\author{
Authors \\ Abdel Jawad Salim Salem*, Eman Yousef Hassan Ayady, Yaser Kamel Hassan Amran \\ *Corresponding Author \\ Abdel Jawad Salim Salem \\ First Specialist in Family Medicine, Prince Hussein Hospital, Jordan
}

\begin{abstract}
The serum of ferritin used as biomarker of different diseases. This paper aimed at studying the possibility of using ferritin on diagnosing diseases. To accomplish that, random sample of subjects included in this research. The results showed that the serum of ferritin can be used as a biomarker of some diseases, while in some cases it did not reflect the disease level. The study recommended that the use of ferritin serum level can be used as first indicator for diseases but still deep diagnosis required to complete the disease level of patients.

Keywords: Serum ferritin, biomarkers, chronic diseases.
\end{abstract}

\section{Introduction}

In recent diagnosis, the conversion to biomarkers widely used to diagnose patients as it facilitates high response for patients' situations ${ }^{[1]}$. Biomarkers are used as disease indicators in the last years. They can be used to indication of disease level. Moreover, biomarkers can be used to assess the disease development overtime and the risk level patients' reached. On the other hand, biomarkers are used to measure the patients' response for treatment and the follow up of changes.

Ferritin level (FL) received high attention as one of the important biomarkers for many diseases ${ }^{[2]}$. The role of ferritin protein with iron metabolism raised its importance as biomarker. The cellular storage of iron molecule is highly related to ferritin protein ${ }^{[3][4][5]}$. The research showed that the normal level of serum ferritin is 13 to 200 $\mathrm{ng} / \mathrm{dl}$. The low level of serum ferritin level reflects the deficiency of iron, which lead to deficiency anemia $^{[5]}$. This research will investigate the possibility of using ferritin in diagnosing different disease through the application on hospital's patients.

\section{Background}

Ferritin protein is responsible for iron metabolism in the body ${ }^{[3][7]}$. The malfunction of ferritin reflects the body disturbances ${ }^{[1]}$. So, different research considered that the serum of ferritin can be a good biomarker for different diseases. Also, it can reflect the level of disease and its stage. The ghaucher disease was predicted using the serum ferritin $^{[2] .}$ The results showed that the serum ferritin gave indication about the disease level. 
Their results showed that the level of serum ferritin decreased significantly in the patient who receive treatment overtime.

In other research, serum ferritin was used to diagnose rheumatoid arthritis ${ }^{[4][5]}$. It was found the biomarker can be used to measure the progress of disease and the level of treatment. The research recommended that using such biomarkers will reduce the medical costs for patients. The use of serum ferritin (SF) to figure out the diabetes has shown that the increase of SF will increase the insulin resistance in patients ${ }^{[8][9]}$.

Using SF as biomarker for breast cancer, the research has shown that the level of SF was higher in breast cancer compared to healthy women ${ }^{[10]}$. The study concluded that the higher concentration of SF level in young women can be used as indication for breast cancer occurrence. Other studies showed positive indication for SF as biomarker on pulmonary hypertension ${ }^{[4]}$, high cell damages in body ${ }^{[5]}$, pneumonia $^{[11]}$.

\section{Methodology}

Retrospective study executed in Prince Hussein Hospital in Jordan to measure the possibility of using ferritin level (>200 ng/dcl) on early diagnosis of diseases. The study executed in the period $1^{\text {st }}$ Jan to 31 of July 2019. The sample of the study included 53 subjects of the hospital patients. The sample composed of males and females with different ages and diseases. Patients welling to participate in this research approved before conducting this research through a special form. Blood samples were collected to measure the level of ferritin level.

On the other hand, the disease history of patient was measured through designed questionnaire distributed on them with the help of nurses at the hospital. The designed questionnaire composed of 14 paragraphs with answers yes/no asking about diseases including:

1. Suffering from kidney failure(dialysis)

2. Any diseases of the liver (viral hepatitis (B or $\mathrm{C}$ )/Iron accumulation in the liver (Hemochromatosis))
3. Thalassemia

4. Inflammation and pain and swelling in the joints of the fingers or toes

5. Inflammation and pain and swelling in the joints of the knees or elbows or wrists or shoulders

6. Stiffness or cramp lasts more than half an hour when waking up in the morning in the neck or back

7. Rheumatoid arthritis

8. Intermittent height and frequent body heat with night sweats and chills at night and loss of appetite with pain in the joints of the body and back

9. Malta fever

10. Colon cancer or breast

11. Sharp decrease in weight during the last six months

12. The breast examination mammogram during the past two years, any illness or malignant tumors.

\section{Results and discussion \\ General description}

The studied sample composed of 52 subjects with equal distribution of males and females (50\% each). The average age of the sample was 56.2 years with standard deviation 14.9. The maximum recorded age was 85 years, while the minimum age was 23 years. The first $25 \%$ of the sample had ages less than 45.5 , while the rest of the sample had ages more than that. The sample subjects visiting Prince Hussein Hospital in Jordan to receive medication for different diseases (Figure 1). The results showed that the number of females with ages less than or equal 60 was lower compared to the males ages. 


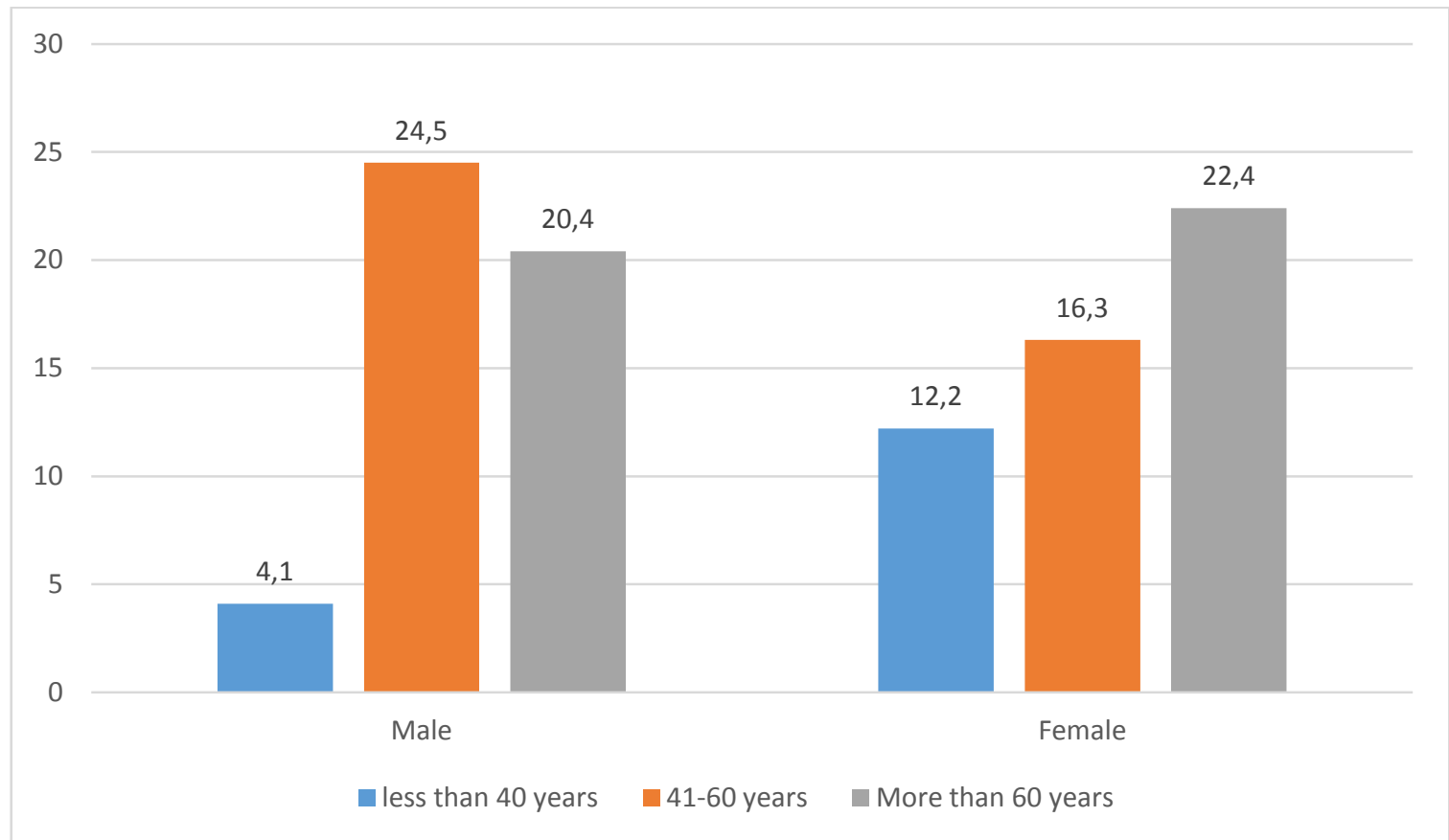

Figure 1: The percentage of age distribution among males and females.

Serum ferritin level varied among the subjects widely. The minimum level recorded was 90.36 ng/dcl, while the highest level recorded was 2000 $\mathrm{ng} / \mathrm{dcl}$. The mean value of the serum ferritin level was $447.8 \mathrm{ng} / \mathrm{dcl}$ with high standard deviation reached 366.75. The ferritin level for more than $70 \%$ of the subjects exceeded the threshold level $200 \mathrm{ng} / \mathrm{dcl}$.

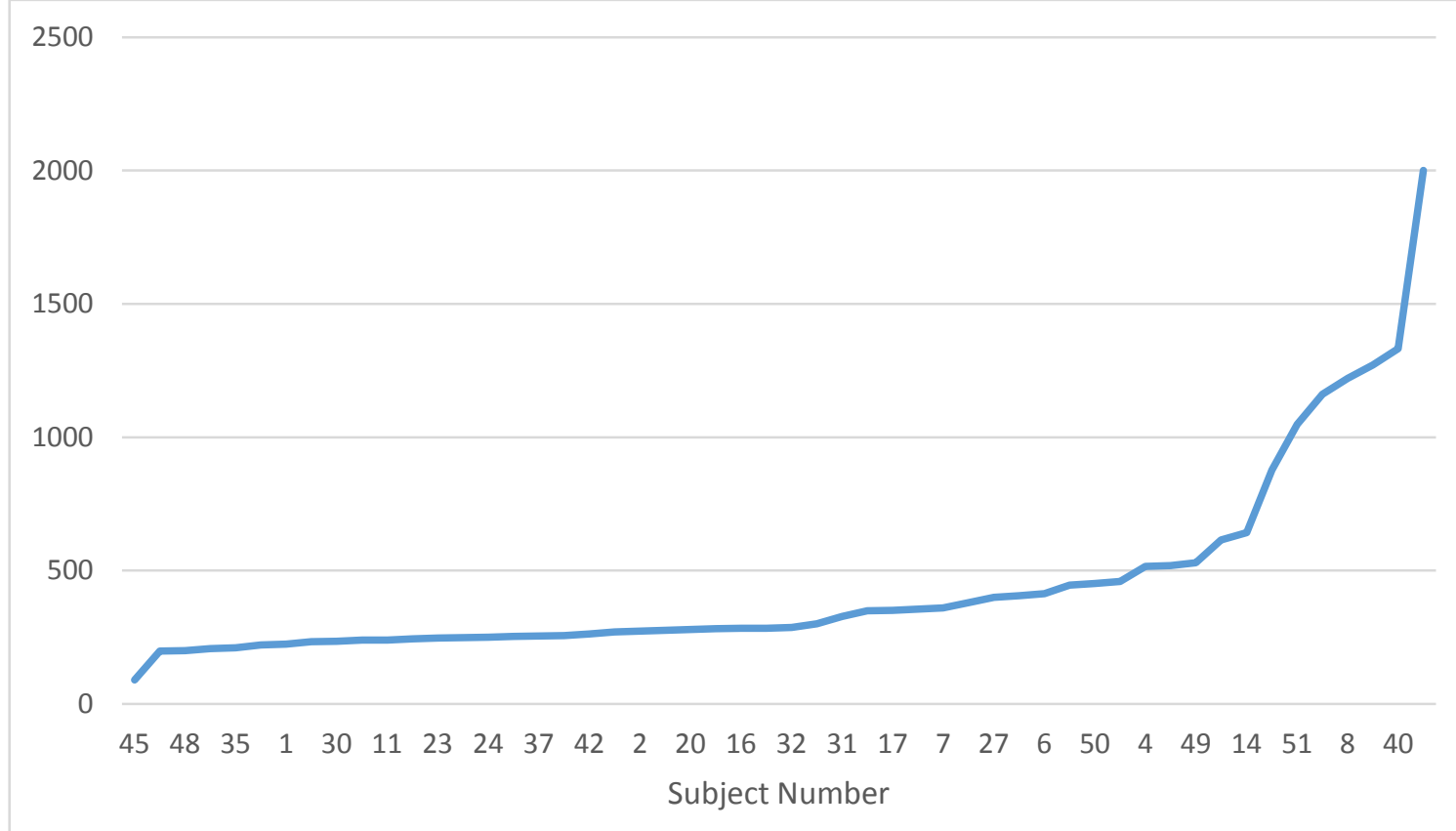

Figure 2: The distribution of serum ferritin level (ng/dcl) among the subjects

\section{Liver Disease}

Figure 3 show the level of SF level at liver diseased patients compared to free patients. The results showed that the level of SF was very high
$1048.9 \mathrm{ng} / \mathrm{dcl}$ compared to non-liver diseased patients. The results were significant at $\mathrm{p}<0.01$. This result indicates the possibility of using the SF as biomarker for liver diseases. 


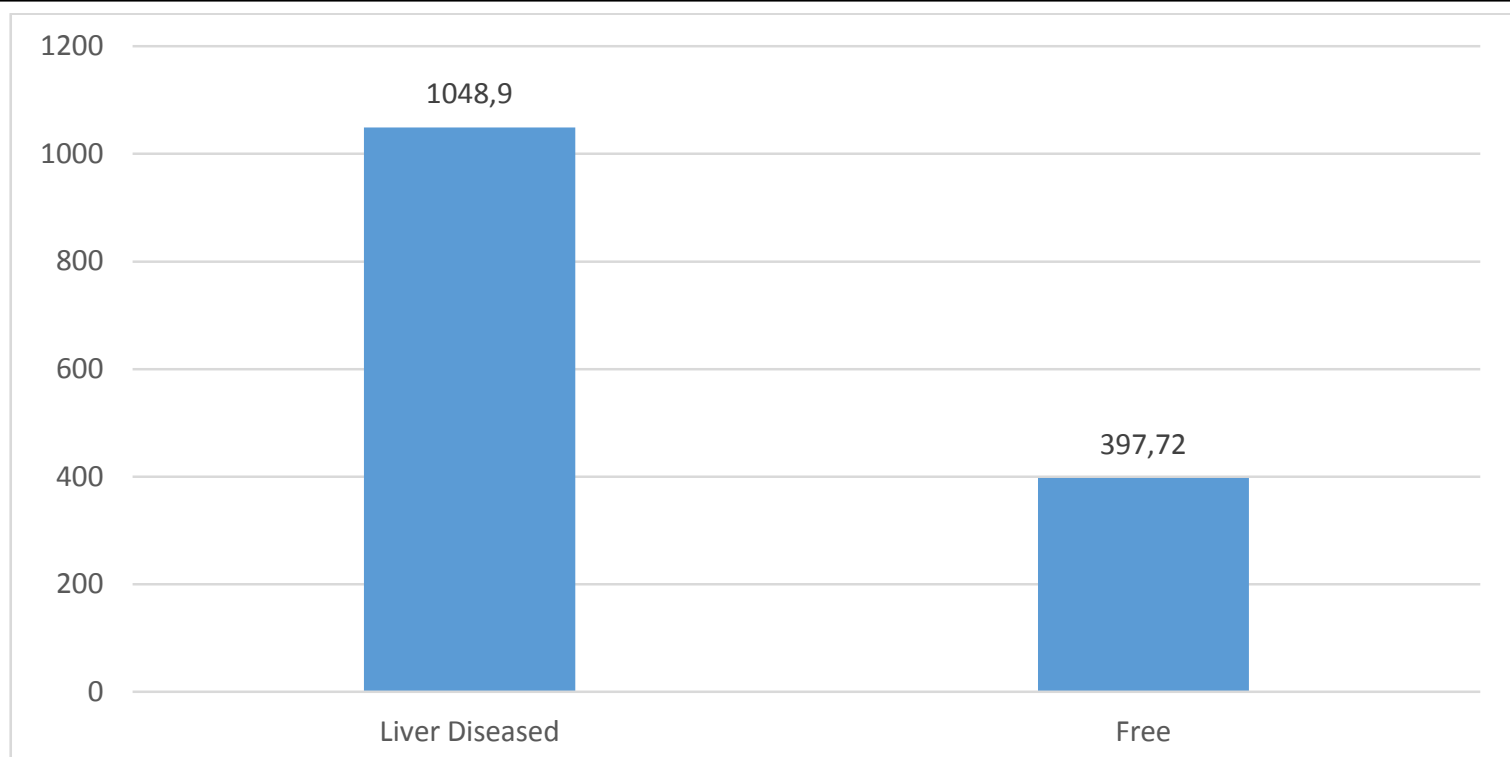

Figure 3: The mean of ferritin serum (ng/dcl) among liver diseased patients compared to free patients $(\mathrm{p}=0.001)$

Contradicted results were found for SF level (Figure 4). The results showed that the patients that do not have family hx thalassemia have high
FS levels compared with patients have this history. This indicates that the patient test of SF does not reflect the family disease history.

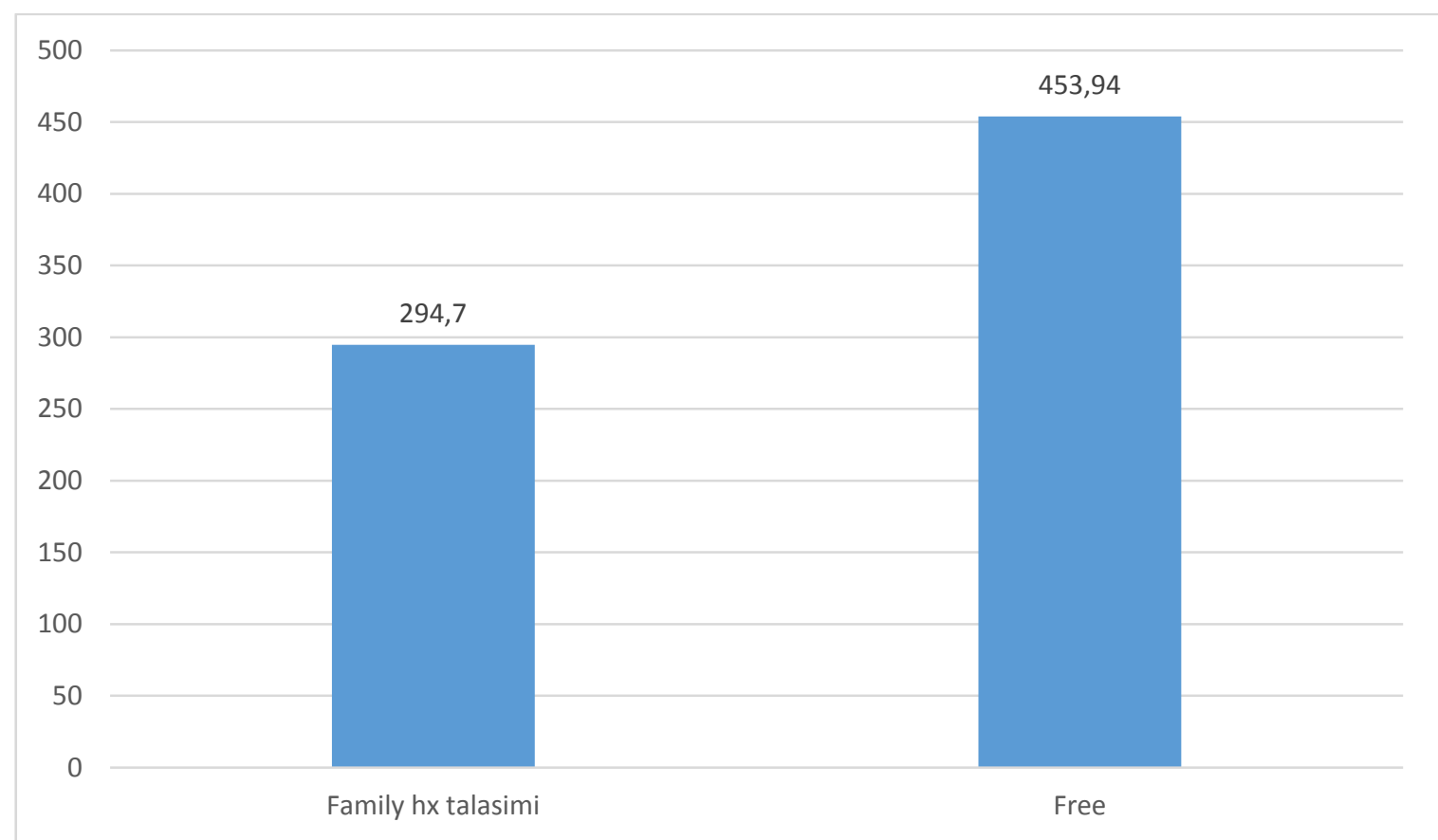

Figure 3: The mean of ferritin serum (ng/dcl) among family hx thalassemia diseased patients compared to free patients $(\mathrm{p}=0.552)$

Concerning blood transferring or iron through IV, the results showed that the patients with blood or iron transfer had higher SF levels compared to other groups without transferring. This may reflects that these patients have medical blood problems reflected through the high SF levels. The compared results were significant at $\mathrm{p}=0.05$ (Figure 4). 


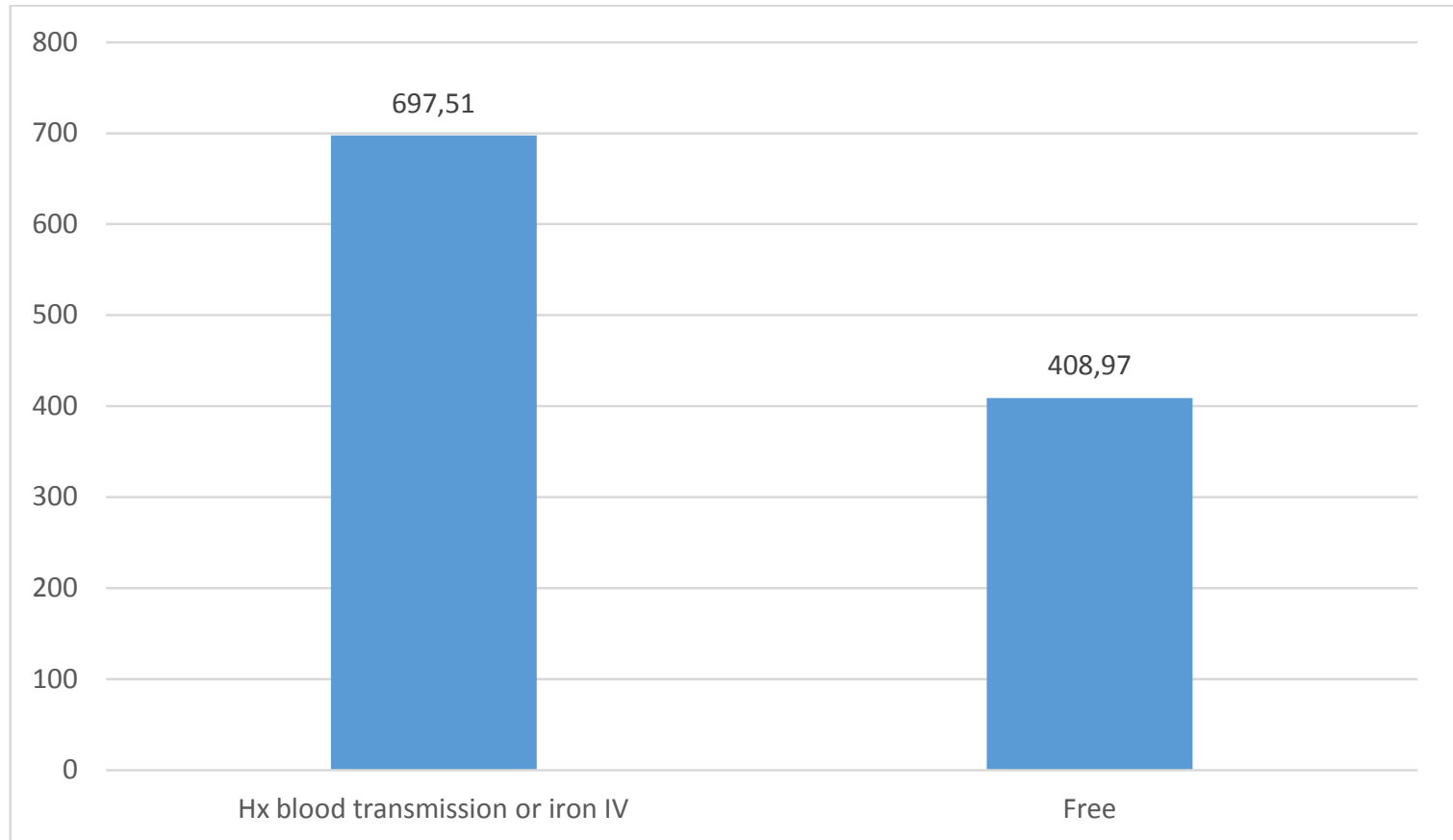

Figure 4: The mean of ferritin serum $(\mathrm{ng} / \mathrm{dcl})$ among blood transferring or iron IV received patients compared to free patients $(\mathrm{p}=0.05)$

Contradicted results were recorded in case Rheumatoid patients with lower level of SF. The results between patients and non-patients were close and the testing showed insignificant different between the two groups.

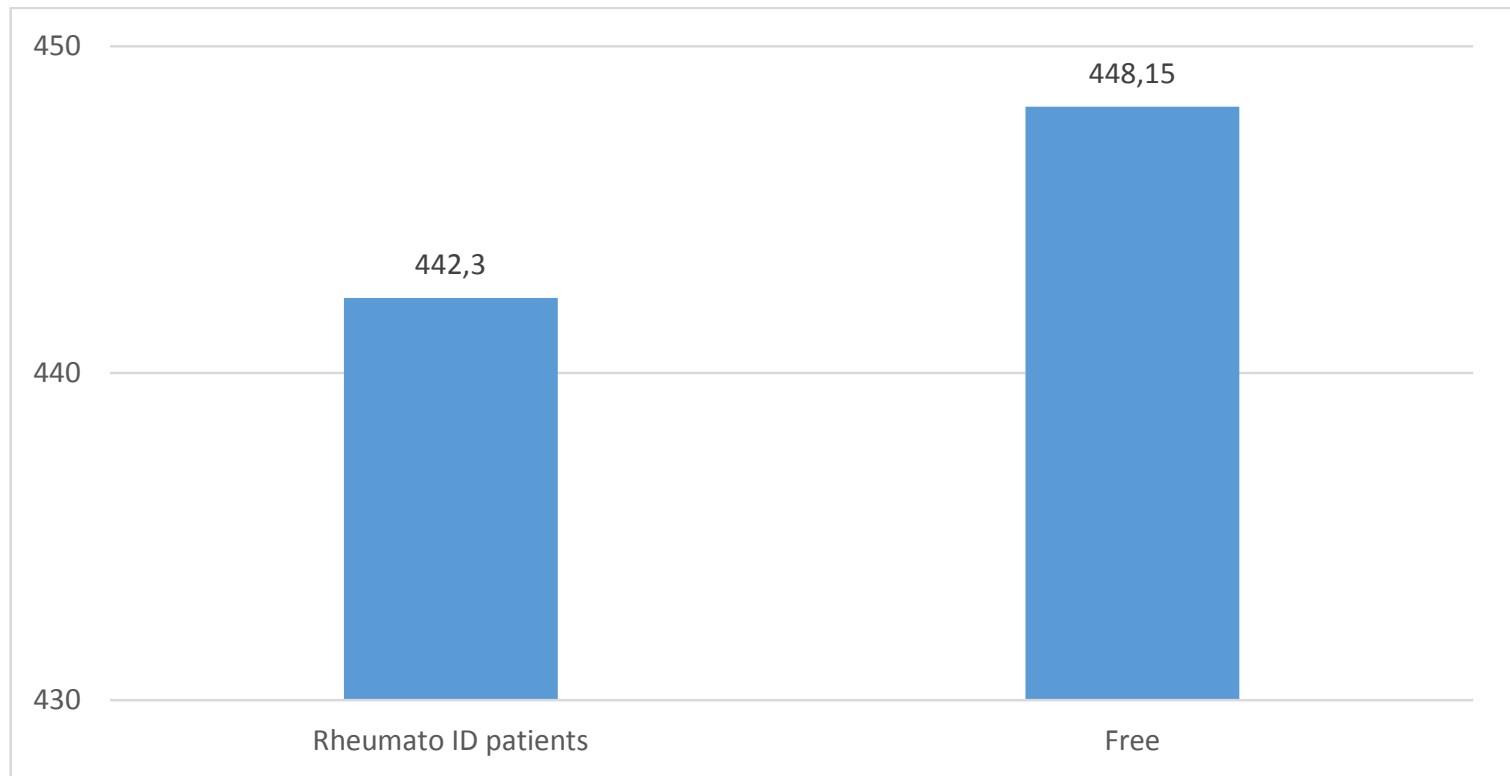

Figure 5: The mean of ferritin serum (ng/dcl) among rheumatic id patients compared to free patients $(\mathrm{p}=0.979)$

The usage of SF level to diagnose the joint pains failed. The results showed that both patients with or without joint pain had close level of SF levels (Figure 6). 


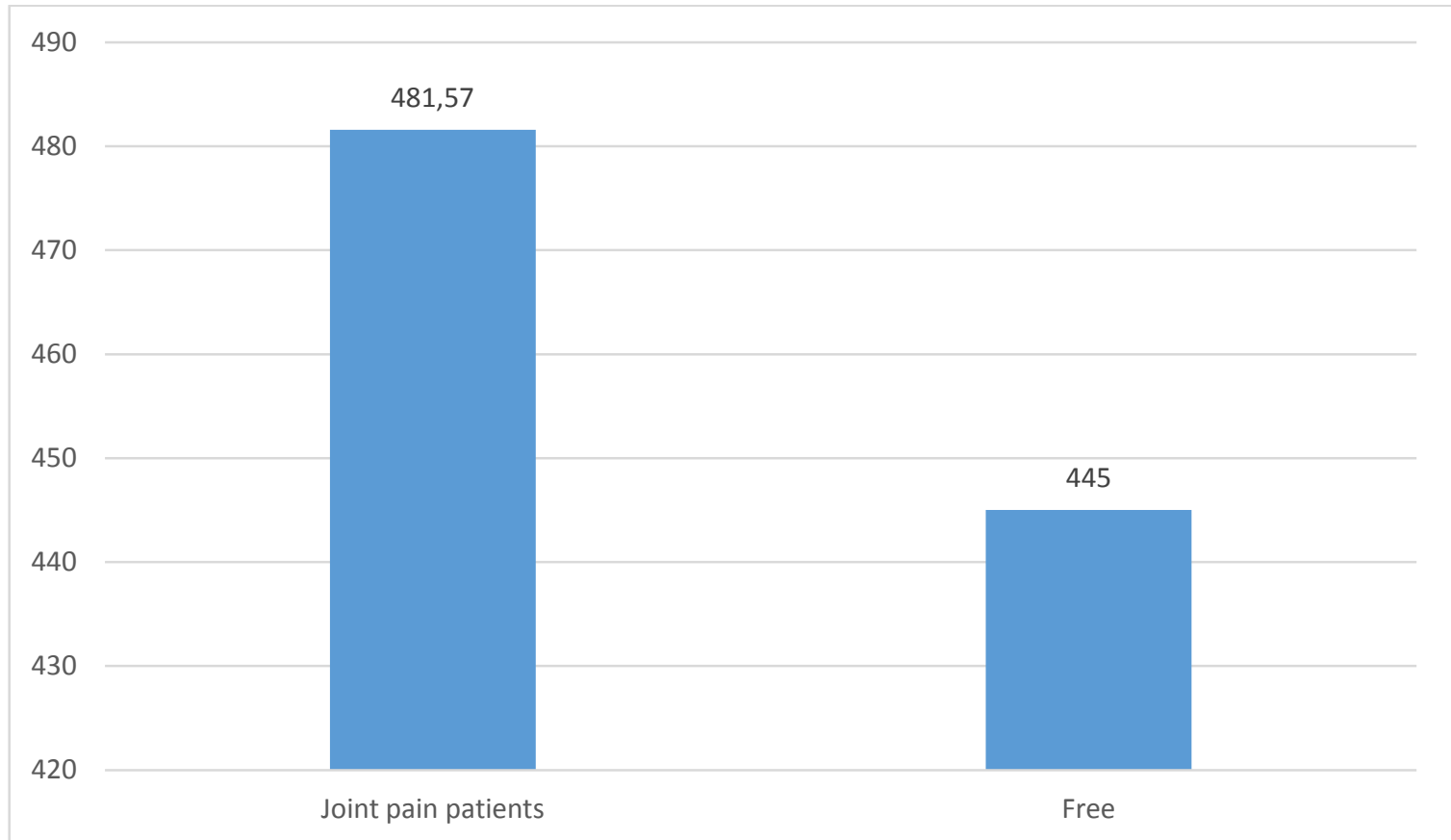

Figure 6: The mean of ferritin serum (ng/dcl) among joints pain patients compared to free patients $(\mathrm{p}=0.85)$

The results showed differences for the diagnosis for cricks among diseased and non-diseased patients (Figure 7). The results showed that the difference between the two groups was not significant despite the high difference between them.

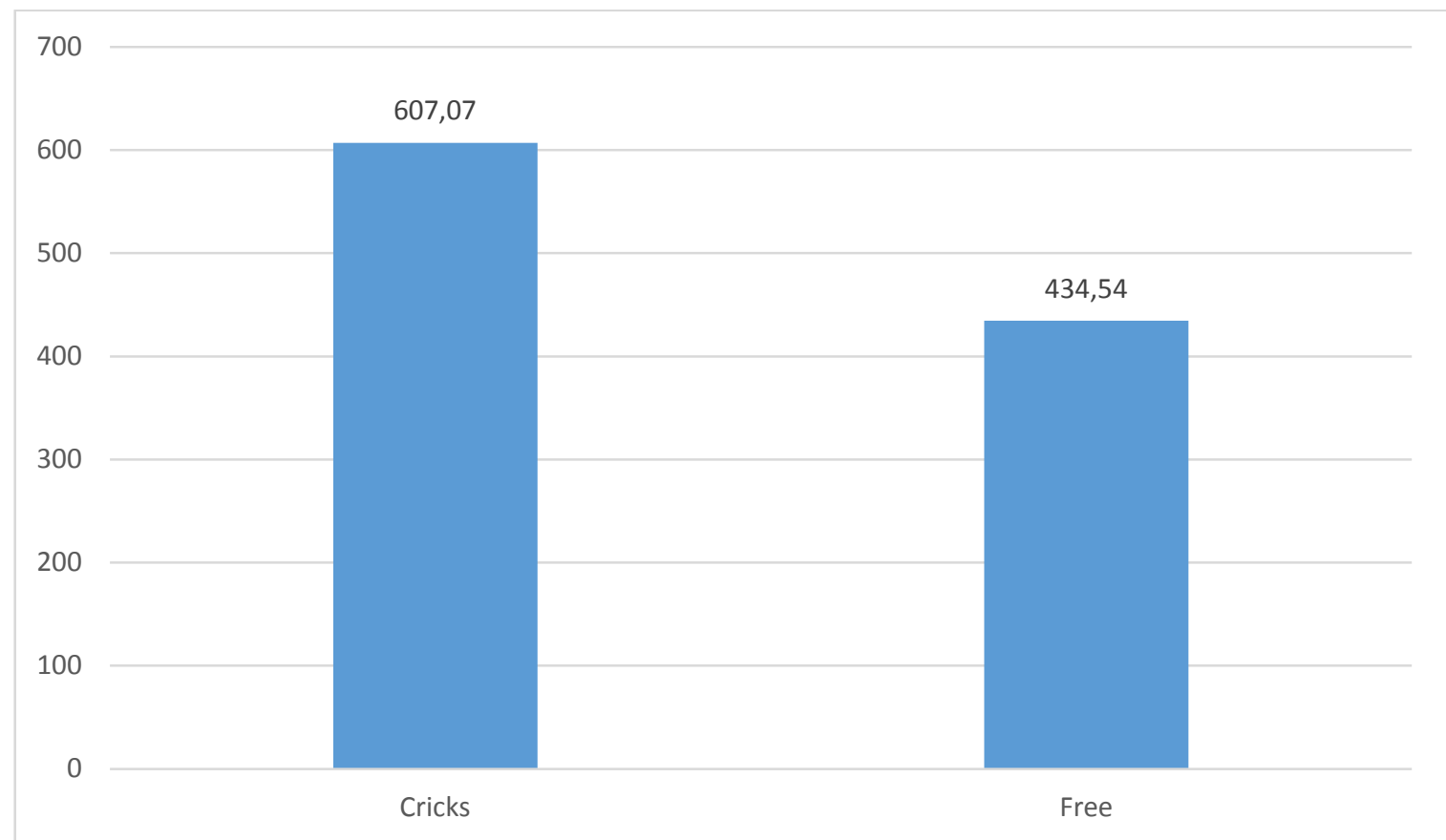

Figure 7: The mean of ferritin serum (ng/dcl) among cricks patients compared to free patients $(\mathrm{p}=0.371)$

The SF level was efficient in diagnosing the polyarthritis rheumatic acuta among patients and non-patients. The results showed significant differences between the two groups $p<0.05$ (Figure 8). 


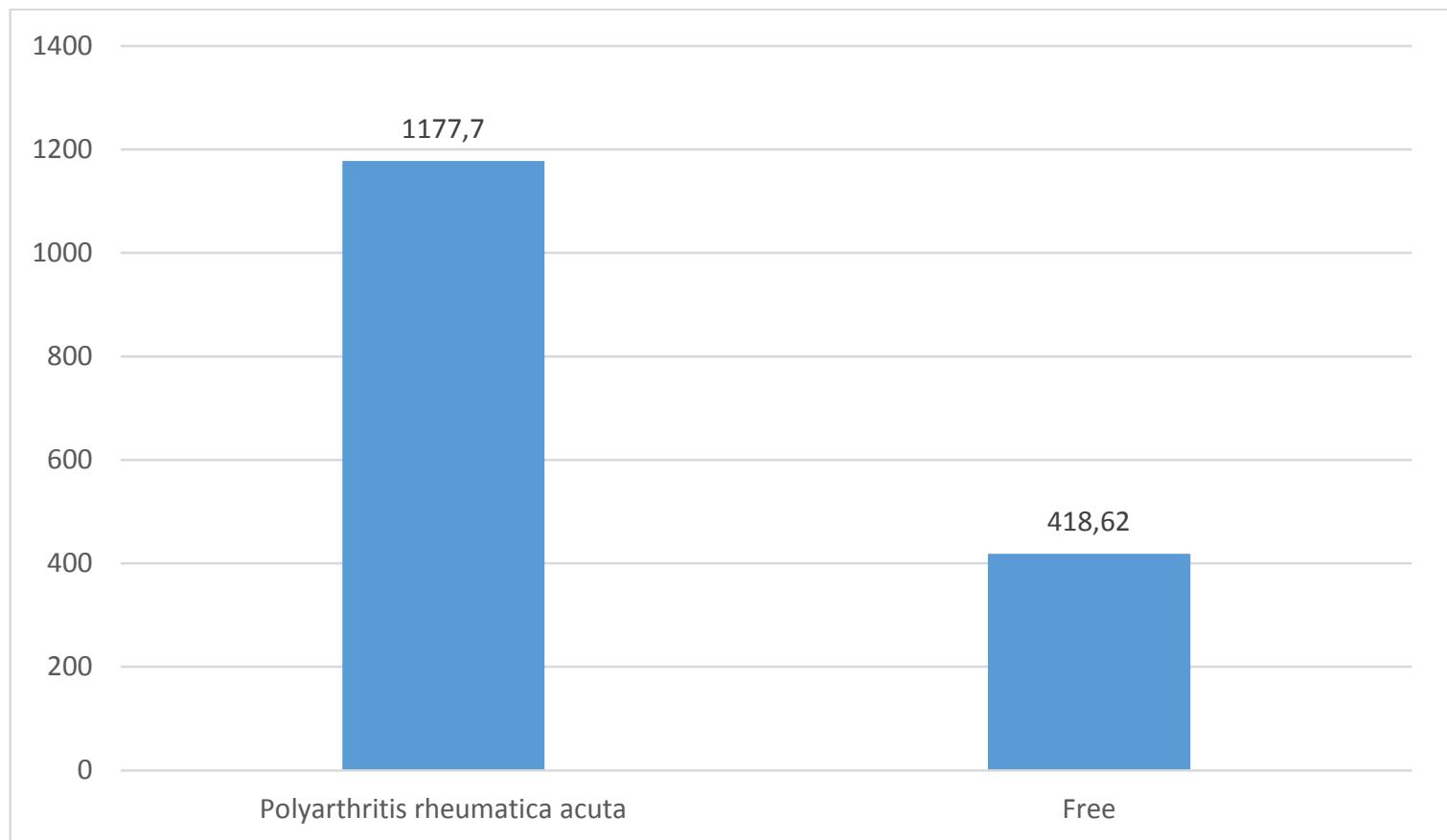

Figure 8: The mean of ferritin serum (ng/dcl) among polyarthritis rheumatic acuta patients compared to free patients $(\mathrm{p}=0.003)$

The biomarker SF level was efficient in diagnosing severe weight loss. The results showed significant differences between the two groups $(\mathrm{p}<0.05)$ (Figure 9).

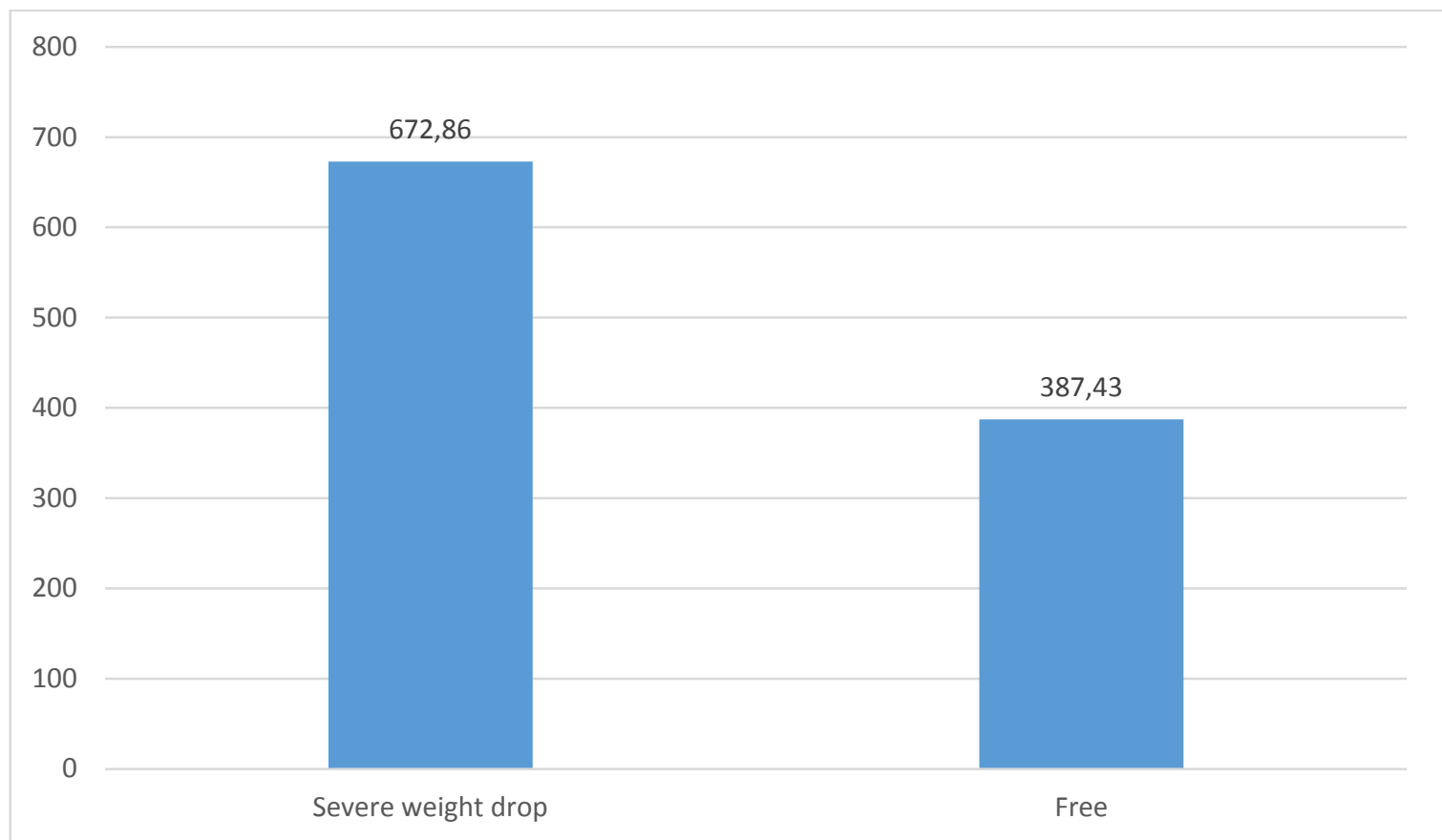

Figure 9: The mean of ferritin serum (ng/dcl) among severe weight loss patients compared to free patients $(\mathrm{p}=0.02)$.

The results showed that SF level biomarker can be used effectively to diagnose the malignant tumors. The results showed significant results for infected patients compared to non-infected patients (Figure $10)$. 


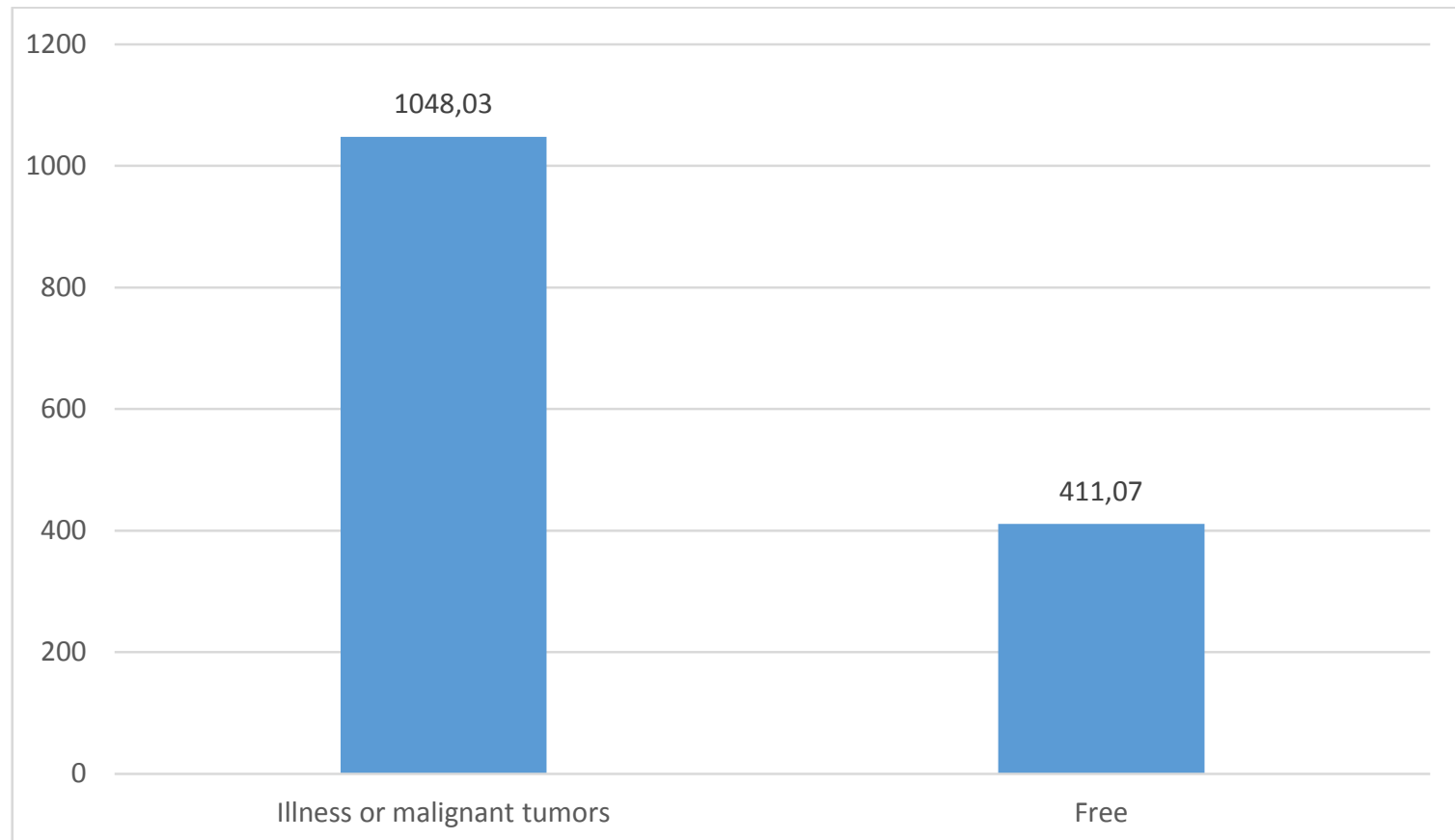

Figure 10: The mean of ferritin serum (ng/dcl) among illness or malignant tumors patients compared to free patients $(\mathrm{p}<.0 .003)$

\section{Conclusions and Recommendations}

The objective of this research is to examine the ability to use SF level as biomarkers for different diseases. The research was applied on a group of hospital patients who participated in this research. The sample included equally females and males and the ages distributed from young to old people. The results showed that some of results were positive while other are negative. The positive results included malignant tumors, blood transferring patients, polyarthritis rheumatic acuta and liver diseases.

\section{References}

1. X. Chen, S. Huang and D. Kerr, "Biomakers in clinical medicine," in Integration of Biomarkers into Epidemiology Study Designs, 2011, pp. 303-322.

2. T. Koppe, D. Doneda and M. e. a. Siebert, "The prognostic value of the serum ferritin in a southern Brazilian cohort of patients with Gaucher disease," Genetics and Molecular Biology, vol. 39, no. 1, pp. 3034, 2016.
3. B. Chlou and J. Connor, "Emerging and dynamic biomedical uses of ferritin," Pharmaceuticals, vol. 11, p. 124, 2018.

4. V. Rameh and A. Kossaify, "Role of biomarkers in the diagnosis, risk assessment, and management of pulmonary hypertension," Libertas Academia: Biomarkers Insights, pp. 85-89, 2016.

5. D. Kell and B. Pretoritus, "Serum ferritin is an important inflammatory disease marker, as it is mainly a leakage products from damage cells," Metallomics, vol. 6, p. 748, 2014.

6. V. Goeb, P. Fardellone, J. Sibilia and F. Ponchel, "Biomarkers in Rheumatoid Arthritis," Mediators of Inflammation , vol. 2014, 2014.

7. M. Knovich, J. Storey, L. Coffman and S. Torti, "Ferritin for the clinician," Blood Rev., vol. 23, no. 3, pp. 95-104, 2009.

8. F. Atzeni, R. Talotta, I. Masala and e. al., "Biomarkers in Rheumatoid Arthritis," IMAJ, vol. 19, pp. 512-516, 2017.

9. S. Gandhi, A. Chaudhari, S. Prantinidhi and A. Sontakke, "Study of serum ferritin 
and $\mathrm{HbA} 1 \mathrm{c}$ in type 2 diabetes mellitus,"

International Journal of Clinical

Biochemistry and Research, vol. 5, no. 4, pp. 594-598, 2018.

10. S. Kashinakunti, K. Hiremath, M.

Rangappa and G. Kallaganada, "Serum ferritin level in type 2 diabetes mellitus: A case control study," International Journal of Clinical Biochemistry and Research, vol. 3, no. 1, pp. 62-66, 2016.

11. D. Cujic, I. Stefanoska and S. Golutbocis, "Serum ferritin in healthy women and breast cancer patients," J Med Biochem, vol. 30, pp. 33-37, 2011.

12. M. Christ-Crain and S. Opal, "Clinical review: The role of biomarkers in the diagnosis and management of community acquired pneumonia," Christ-Crain and Opal Critical Care, vol. 14, p. 203, 2010.

13. B. Lorcerie, S. Audia, M. Samson and e. al., "Diagnosis of hyperferrritinemia in a routine clinical practice," Presse Med., 2017.

14. E. Nassir, "Detection of serum ferritin in women with breast cancer," Iraqi J Pharm Sci, vol. 25, no. 1, pp. 23-27, 2016. 